drugs were used concurrently but no patient received other benzodiazepines. The dosage of nitrazepam in the 6 fatal cases was significantly higher and approximately double that in 22 survivors of the same age range (mean $0.7 \mathrm{mg} / \mathrm{kg} / \mathrm{d}$ ). Patients in both groups had multiple seizures, including generalized tonicclonic, myoclonic and focal types but none had infantile spasms or absence attacks. Perinatal asphyxia, Prader-Willi syndrome, pertussis vaccine-related encephalopathy, and nonketotic hyperglycinemia were the etiological diagnoses in 4 of the 6 fatal cases. Three of the 6 had known factors contributing to death: congestive heart failure, aspiration of gastric contents, and unexplained hyperthermia, shock, and respiratory failure. Three patients died unexpectedly and autopsies in 2 were unrevealing. The cause of death was undertermined but a nitrazepam-induced swallowing disturbance and aspiration was suspected. The authors recommend that the use of nitrazepam in young children should be restricted to those resistant to other antiepileptic drugs, the dose should not exceed $0.8 \mathrm{mg} / \mathrm{kg} / \mathrm{d}$, and children with prior swallowing difficulties should be observed closely. (Murphy JV et al. Deaths in young children receiving nitrazepam J Pediat 1987; 111:145-147).

COMMENT: Nitrazepam (Mogadon) is considered the most effective benzodiazepine for control of infantile spasms and other myoclonic seizures. It is not usually recommended for the treatment of generalized tonic-clonic seizures, as employed in this study, which might explain the necessity for the larger doses in the affected infants.

In a study at Children's Memorial Hospital, Chicago (Millichap JG, Ortiz WR. Am J Dis Child 1966; 112: 242), the side effects of nitrazepam in a trial involving 36 infants and children with myoclonic seizures included drowsiness (50\%), ataxia (20\%), hypotonia (20\%), and muscular weakness (9\%). Anorexia and vomiting developed in $2 \%$ and skin rash in $2 \%$. the most serious adverse effects of nitrazepam in our study were symptoms suggesting autonomic dysfunction, previously unreported. Excessive drooling of saliva occurred in $9(25 \%)$ infants and pulmonary congestion with wheezing developed in 4 ( $11 \%$ ) debilitated infants with diplegia and severe retardation. Miosis of the pupils was also noted. The pulmonary symptoms required withdrawal of the drug or reduction of the dose to less effective levels and possible fatalities were avoided. The nitrazepam-induced drooling and aspiration in some cases have recently been explained by a delay of cricopharnygeal relaxation (Wyllie $\mathrm{E}$ et al. N Engl J Med 1986; 314:35-38). The tolerance that develops to nitrazepan and the autonomic and sedative side-effects of larger doses seriously detract from the usefulness of this agent in long-term theraphy of infantile spasms. In debilitated infants, perhaps nitrazepam should be contraindicated.

\title{
WITHDRAWAL OF ANTIEPILEPTIC TREATMENT
}

Investigators at the Division of Neurology and Clinical Neurophysiology, Hospital General de Catalunya, Barcelona, Spain, evaluated the withdrawal of antiepileptic treatment over a one year period in 608 epileptics who had been seizure-free for 5 years. Relapses occurred in 144 (23.7\%) of the total group. Among 474 patients in whom details of seizure types were available, relapses occurred in 119 (25.1\%) of the total. Idiopathic generalized epilepsies showed a $19.6 \%$ relapse reate whereas symptomatic generalized epilepsies relapsed in $37.9 \%$ of patients. The lowest relapse rate occurred in patients with absence seizures $(9.9 \%)$ and the highest rates in those with west 
( $50 \%$ ) and Lennox-Gastaut ( $43.7 \%$ ) syndromes. Grand mal and complex partial seizures recurred in $27.8 \%$ and $23.3 \%$ of patients, respectively. The majority of relapses were single isolated seizures and occurred in the first year after drug withdrawal.

Risk factors for seizure relapse included 1) delay in initiating anticonvulsant therapy, 2) a symptomatic etiology, 3) mixed seizure types, 4) status epilepticus occurrence, and 5) signs of permanent neurologic damage. (Oller-Daurella L, Oller, F-V L. Suppression of antiepileptic treatment. Eur Neurol 1987 ; $\underline{27}: 106-113$ ).

COMMENT: The 5 year seizure-free period before drug withdrawal in this study is longer and approximately twice that employed by other investigators. Shinnar S et al (N Engl J Med 1985; 313:976) reported the same 25\% relapse rate among $88 \mathrm{child}$ ren who had been seizure free for only 2 years.

The withdrawal of antiepileptic medication should not be determined by a set seizure-free time-period for all types of epilepsy. Each patient should be evaluated individually, having regard to several factors, including psychological, predictive of potential relapse. Withdrawal is probably contra-indicated or likely to be unsuccessful in patients with the following: 1) symptomatic epilepsies with radiological or neurological evidence of structural cerebral defect, mixed seizure types, Lennox-Gastaut syndrome, focal seizures, complex partial with secondary generalized tonic-clonic patterns, 3) history of status epilepticus and 4) psychological stress, especially in adolescents and young adults.

Th prognostic value of the EEG is controversial; these authors and Thurston JH et al ( $N$ Engl J Med 1982; 306:831) found a negative correlation whereas others regard the EEG as an important factor, predictive of a good outcome when normal (Shinnar S et al 1985). An abnormal EEG was one of the most significant predictors of relapse after drug withdrawal in the study by Emerson R et al ( $\underline{N}$ Engl J Med 1981; 305:1125).

\section{CARBAMAZEPINE AND COGNITIVE IMPAIRMENT}

Members of the Departments of Pediatrics, Neurology, and Clinical Pharmacology and Toxicology at the Children's Hospital and Ohio state University, Columbus, OH 43205 , assessed neuropsychological function before and after carbamazepine monotheraphy at low $(<-7.5, \mathrm{ug} / \mathrm{ml})$ and moderate $(>8.0, \mathrm{ug} / \mathrm{ml})$ plasma levels in 11 children ( 4 boys 7 girls, mean age 9.8 yrs) with controlled complex partial epilepsy. Carbamazepine caused significant impairments $(P<.03)$ or efficiency in learning of new information (Paired Associates Test) and short-term memory scanning (Sternberg Memory and Reaction Time Paradigm) that were associated with moderate plasma concentrations within the therapeutic range. The decline in performance was not accompanied by a greater abnormality on the EEG or carbamazepineinduced seizure exacerbation (see Pediatric Neurology Briefs, Vol 1, No 1). A mild beneficial effect on speeded eye-hand coordination was suggested at moderate plasma levels but only in the nonpreferred hand. Except for a trend toward more rapid memory scanning, there was no change in performance from the baseline to low drug level assessment. (O'Dougherty M, Wright FS et al. Carbamazepine plasma concentration. Relationship to cognitive impairment. Arch Neurol 1987; 44:863-867).

COMMENT: These results are in agreement with previous studies in adults that have shown impairments in concentration and memory-processing with higher 